\title{
CLASSIFICAÇÃO DO USO/COBERTURA DA TERRA POR MEIO DE ALGORITMOS NÃO PARAMÉTRICOS COMPARADOS AO CLASSIFICADOR DE MÁXIMA VEROSSIMILHANÇA
}

\author{
LANDUSEILANDCOVER CLASSIFICATIONBY NON-PARAMETRIC \\ ALGORITHMS COMPARED TO THE LIKELIHOOD CLASSIFIER
}

\author{
Thomaz Corrêa e Castro da Costa ${ }^{1}$ \\ Paulo De Marco Júnior ${ }^{2}$ \\ Ricardo Seixas Brites ${ }^{3}$
}

\begin{abstract}
RESUMO
Mapas de uso/cobertura da terra gerados por meio de estratégias de classificação não paramétrica de uma imagem Landsat TM foram comparados ao resultado gerado pelo tradicional classificador de máxima verossimilhança (MAXVER). Algumas estratégias não paramétricas, desenvolvidas neste trabalho, tiveram a finalidade de diminuir a limitação do classificador não paramétrico de Skidmore/Turner, que requer um tamanho de amostra de treinamento muito grande, comparado ao MAXVER, para reduzir a área não classificada na imagem. Os resultados mostraram a superioridade do MAXVER, na combinação entre exatidão e área não classificada na imagem. De qualquer forma, a classificação não paramétrica tem a propriedade de não necessitar da pressuposição de normalidade dos dados, utilizando a real informação das amostras de treinamento para classificação, o que lhe assegura maior exatidão.

Palavras-chave: Uso/Cobertura da Terra, algoritmo não paramétrico, classificador de Skidmore/Turner, Landsat TM, sensoriamento remoto.
\end{abstract}

\footnotetext{
1 Embrapa Solos, R. Jardim Botânico, 1.024 CEP 22460-000, Rio de Janeiro, RJ thomaz@cnps.embrapa.br

2 Depto Ecologia, Universidade Federal de Viçosa-UFV, CEP 36571-000, Viçosa, MG pdemarco@ufv.br

3 Universidade Católica de Brasília-UCB, CEP 70790-160, SGAN 916 - Módulo B - Asa Norte - Brasília - DF rbrites@pos.ucb.br
} 


\section{ABSTRACT}

Landuse/landcover maps produced by non-parametric classifiers of a Landsat TM image were compared with results obtained by the usual likelihood classifier (MAXLIKE). The maximum likelihood classifier is the parametric strategy pixel the pixel most used in orbital images classification. But Skidmore and Turner (1988) obtained results of global accuracy with their algorithm non parametric in SPOT XS data, for classes of Pinus spp. age superior $14 \%$ in relation to the algorithm of maximum likelihood. The algorithm of Skidmore/Turner suffered modification proposal in Lowell (1989), Gong and Dunlop (1991) and Dymond (1993). The purpose of the algorithm non-parametric that were developed in this work was to diminish the limitation of the Skidmore/Turner non-parametric classifier that, compared to MAXLIKE, demands a very large training sample to reduce the not classified areas in the image. This algorithm, a new supervised nonparametric classifier that make flexible the association amidst brightness values was developed for testing the assumption that increases the image classified area without missing accuracy and for solving the limitation of the nonparametric classifier of Skidmore/Turner, that requires a larger training sample for reducing the of unclassified pixels in the image. To facilitate the understanding of the algorithms used in this work, a simplified example of data of remote sensing is presented, which corresponds to a window of image of $20 \times 20$ pixels, with two classes sampled: lagoon and forest, according to the training samples in Table 1, extracted from 3,4 and 5 Landsat TM bands. The main difference of the nonparametric classifier of Skidmore/Turner in relation to MAXLIKE is in $p(X \mid)$ calculation. The training samples to that classifier are constituted of discrete groups of pixels vectors, common to each class, and no more a continuous space determined by the multivariate normal function. For the purpose of reducing the restrictive effect of non-parametric of Skidmore/ Turner, NPVIC it was developed a supervised non-parametric algorithm, by pixel, which had on principle to make flexible the rigid association among brightness values of the pixel vector. This algorithm developed from the conditional probability $P(i \mid X)$, considers the brightness values independent for band and attributes to the pixel the class that integrates the maximum value of intersections by bands, pondered by the sizes of the classes. The area is placed in the microregion of Viçosa, Minas Gerais, Brazil, with predominance in this municipal district. Part of an image was used of $15 \times 15 \mathrm{~km}(22.500 \mathrm{ha})$, extracted from the Landsat TM image, 217/74, South Quadrant, of 10/10/94, composed of TM 3, 4 and 5 band. Eleven (11) classes of landuse and landcover were defined, described in Table 8. The reference sample was constituted by a systematic grid of 163 points, distant of 28 pixels on the area of the photos, more 146 intermediary points among the points of the bars (total of 309 points). The index used to evaluate the results of the classifications was the global accuracy (G). The non-parametric algorithm of Skidmore/Turner, the non-parametric algorithm of Skidmore/Turner "modified" by Dymond, NPVIC, and NPVIC with of Dymond's normalization (Apendix) were written in-house software in Pascal Language, utilizing modules of selection of training samples of the IDRISI 2.0 for Windows (vectorization Screen and MAKESIG). The algorithm of maximum likelihood used was MAXLIKE of the same software. The results of global accuracy and the $\%$ of the classified area in the image are presented in Table 9 and the Figure 4 shows the ranking of results of $\mathrm{G}$ and \%ANC to all the classifiers and strategies applied to the compression factors and original data, as well as the test $Z$ of seqüencial significance among the results $1 \%$. The combination of the best accuracy results with the smallest percentages of non-classified area occurs to the algorithm of maximum likelihood with options 0 and $1 \%$. The largest levels of \%ANC are represented by the algorithms NPSKID, NPSKIDYM and NPVICB3. And the smallest indexes of accuracy (G) are represented by the algorithms NPVICA1 and NPVICDYMA1. The non-parametric NPVIC algorithm allows to reduce the non-classified area in an image, when compared to NPSKID, but not in a satisfactory way, since for classifying the whole area images of low accuracy. The Skidmore/Turner's non-parametric algorithm to sizes of training sample sufficient to MAXLIKE, produces classifications of low quality. The results showed the superiority of MAXLIKE regarding accuracy and not classified area in the image. In any way, non-parametric classification does not need the presupposition of normality of data hence it uses real information from training samples for classification that ensures bigger accuracy.

Key-words: landuse/landcover, non-parametric algorithm, Skidmore/Turner classifier, Landsat $\mathrm{TM}$, remote sensing. 
COSTA, T. C. C. et al. Classificação do uso/cobertura da terra por meio de...

\section{INTRODUÇÃO}

O classificador de máxima verosimilhança (MAXVER) é a estratégia paramétrica mais utilizada em classificação do Uso/Cobertura da Terra por meio de imagens orbitais. Isto se verifica pela sua robustez, no sentido de que a exatidão gerada não é muito sensitiva, tanto a violações moderadas quanto severas da pressuposição de multinormalidade dos dados de treinamento (Swain \& Davis 1978). Em contribuição a sua perfor-mance está o fato de que a maioria das feições seguem distribuição multinormal em imagens de sensoriamento remoto, com grande concentração de dados em torno da média, e freqüências de dados decrescentes nos extremos, que representam o aumento gradual de mistura com outras feições.

Por outro lado, a investigação de classificadores não paramétricos visa obter melhores índices de exatidão para classificações do Uso/Cobertura da Terra (Skidmore \& Turner 1988, Kloer 1994, Cortijo \& La Blanca 1997). O uso destes classificadores torna desnecessária a pressuposição de normalidade dos dados de treinamento, pois segundo Cortijo e La Blanca (1997) a classificação gerada pelo MAXVER é de baixa exatidão quando ocorre grande superposição entre os dados de treinamento no espaço $\mathrm{n}$-dimensional formado por $\mathrm{n}$ bandas.

Skidmore e Turner (1988) obtiveram resultado de exatidão global com seu algoritmo não paramétrico superior em $14 \%$ com relação ao MAXVER em dados SPOT XS, para classes de idade de pinus. Seu classificador utiliza distribuições multifreqüência verdadeiras das amostras de treinamento, e não distribuições multinormais ajustadas como o MAXVER. Este algoritmo sofreu proposta de modificação em Lowell (1989), Gong e Dunlop (1991) e Dymond (1993).

Lowell propôs uma alteração de pouca importância no algoritmo de Skidmore/Turner para solucionar o problema de erro locacional para classes de uso da terra que apresentam maior mistura, pois não observou as limitações desta estratégia para dados reais de sensoriamento remoto.

Para dados Landsat TM por exemplo, com resolução radiométrica de 256 níveis de valores de brilho, e um grande número de classes para classificar em uma cena, as tendências de estimativa de área total seriam diluídas pelo número de classes, e o erro locacional (estimativa individual, do pixel) iria aumentar, pois a classe de maior pertinência neste método não é atribuída deterministicamente, mas aleatória, utilizando-se dos intervalos de probabilidade, determinados pela geração de números aleatórios (Costa 1999).

A contribuição de Gong e Dunlop (1991) ao algoritmo de Skidmore/Turner foi principalmente com respeito à œrreção da função apresentada em Skidmore e Turner (1988). Os erros de notação permaneceram na errata em Lowell (1989), causando confusão na sua interpretação. Sua outra contribuição foi a proposta de modificação na função, eliminando seu denominador que é constante para todas as classes em um pixel. Esta estratégia, que já é adotada na função de máxima verosimilhança, permite uma melhoria no desempenho computacional do classificador.

A modificação proposta por Dymond (1993), mais importante, abordou a ponderação da evidência de um pixel pertencer a uma determinada classe. Originalmente esta ponderação é feita pelo tamanho da classe (número de pixels da classe coletados pela amostra de treinamento). No algoritmo proposto por Dymond esta ponderação é feita pela freqüência média da classe, excluindo-se os valores que não ocorrem (freqüência zero). A freqüência média da classe é o tamanho da classe dividido pelo número de valores distintos na classe. A inclusão desta nova normalização na regra de decisão aumenta a chance para classes de maior variabilidade, pois o tamanho da classe é proporcional ao desvio-padrão do histograma n-dimensional (Dymond 1993).

No presente artigo foi desenvolvido um classificador Não Paramétrico para solucionar o problema de pixels não classificados na imagem, uma limitação inerente ao classificador não paramétrico de Skidmore/Turner. Este classificador, denominado de NPVIC, uma referência à Cidade de Viçosa (MG), não utiliza o conceito de espaço n-bandas, mas desenvolve uma regra de decisão que integra as decisões de distintas bandas, de forma seme- 
Ihante ao classificador do paralelepípedo, com a diferença de utilizar dados reais e não um parâmetro da distribuição como limites inferior/superior ou desvio-padrão da classe.

Assim, o objetivo deste trabalho foi testar o NPVIC, comparando-o com: (1) MAXVER; (2) classificador não paramétrico de Skidmore/Turner; e (3) classificador não paramétrico de Skidmore/Turner "melhorado" por Dymond (1993), avaliando o índice de exatidão e percentagem de área não classificada, com o intuito de investigar a eficiência publicada dos classificadores não paramétricos em comparação ao MAXVER, nos seguintes aspectos: esforço amostral de treinamento, exatidão e área classificada na imagem.

\section{DESCRIÇÃO DOS ALGORITMOS POR MEIO DE UM EXEMPLO}

Para facilitar a compreensão dos algoritmos utilizados neste trabalho apresentamos um exemplo simplificado de dados de sensoriamento remoto, que corresponde a uma janela de imagem de $20 \times 20$ pixels, com duas classes amostradas: Lagoa e Floresta,

Quadro 1 - Vetores de Pixels amostrados para Lagoa e Floresta nas Bandas 3, 4 e 5 do Landsat5-TM. Vectors of sampled pixels for lagoon and forest in bands TM 3, 4 and 5 of the Landsat5-TM.

\begin{tabular}{cccc|c|ccc}
\hline LAGOA & & VB & & FLORESTA & \multicolumn{3}{|c}{ VB } \\
\hline PIXEL & B3 & B4 & B 5 & PIXEL & B3 & B4 & B5 \\
\hline 30 & 11 & 4 & 2 & 7 & 14 & 25 & 37 \\
18 & 11 & 6 & 2 & 2 & 15 & 38 & 45 \\
70 & 11 & 6 & 2 & 3 & 15 & 47 & 41 \\
22 & 11 & 6 & 2 & 35 & 16 & 48 & 36 \\
29 & 12 & 4 & 2 & 6 & 15 & 49 & 50 \\
14 & 17 & 5 & 1 & 11 & 15 & 54 & 47 \\
31 & 12 & 5 & 1 & 32 & 15 & 58 & 42 \\
25 & 12 & 5 & 4 & 1 & 16 & 39 & 50 \\
5 & 12 & 6 & 0 & 14 & 16 & 51 & 51 \\
19 & 12 & 6 & 0 & 1 & 16 & 52 & 10 \\
21 & 12 & 6 & 0 & 33 & 16 & 56 & 43 \\
32 & 12 & 6 & 0 & 25 & 16 & 57 & 45 \\
3 & 12 & 6 & 1 & 26 & 16 & 58 & 16 \\
4 & 12 & 6 & 1 & 22 & 16 & 59 & 48 \\
17 & 12 & 6 & 2 & 16 & 16 & 59 & 50 \\
27 & 12 & 6 & 1 & 13 & 16 & 62 & 50 \\
13 & 13 & 5 & 0 & 34 & 17 & 53 & 40 \\
24 & 13 & 5 & 0 & 24 & 17 & 55 & 47 \\
10 & 13 & 5 & 2 & 17 & 17 & 56 & 48 \\
11 & 13 & 5 & 2 & 31 & 17 & 58 & 42 \\
12 & 13 & 5 & 2 & 10 & 17 & 58 & 46 \\
15 & 13 & 5 & 2 & 27 & 17 & 58 & 46 \\
23 & 13 & 5 & 2 & 23 & 17 & 58 & 46 \\
6 & 13 & 6 & 0 & 30 & 17 & 59 & 43 \\
7 & 13 & 6 & 1 & 78 & 17 & 59 & 45 \\
7 & 13 & 6 & 1 & 29 & 17 & 59 & 49 \\
1 & 13 & 6 & 2 & 15 & 17 & 60 & 48 \\
9 & 13 & 6 & 2 & 19 & 17 & 60 & 49 \\
26 & 13 & 6 & 3 & 5 & 17 & 61 & 18 \\
28 & 13 & 6 & 3 & 20 & 17 & 62 & 50 \\
16 & 14 & 6 & 0 & 21 & 17 & 54 & 49 \\
8 & 11 & 6 & 1 & 5 & $1 B$ & 54 & 46 \\
& & & 9 & 18 & 57 & 50 \\
& & & 12 & 18 & 60 & 48 \\
& & & & 18 & 19 & 60 & 16 \\
\hline & & & & & & &
\end{tabular}


conforme os dados de treinamento no quadro 1, extraídos das bandas 3, 4, 5 do Landsat5TM. Foram amostra-dos 32 pixels de Lagoa e 35 pixels de Floresta.

\section{O Classificador de Máxima Verosimilhança (MAXVER)}

O MAXVER classifica individualmente cada pixel, necessita de amostras de treinamento para classificar a imagem e trabalha com distribuições Gaussianas ajustadas a partir das estatísticas obtidas da amostra de cada classe (classificador pixel-a-pixel; supervisionado e paramétrico). A tomada de decisão em se atribuir uma determinada classe a um pixel, de acordo com sua evidência, se baseia na minimização média de perdas, conhecida também como estratégia de otimização de Bayes (Swain \& Davis 1978). Considera-se como função de perdas a atribuição de um custo ou prejuízo quando se toma uma decisão errada.

Em classificação de imagens uma função de perda é aquela que atribui um valor (prejuízo) quando um determinado pixel é da classe $j$ mas foi classificado como da classe $i$. Assumimos como notação $\lambda(\mathrm{ilj})$, sendo $i, j=1,2, \ldots$, m classes. A estratégia básica é minimizar a perda média na execução da classificação envolvendo todas as classes. Para um pixel $X$, a perda média resultante da decisão de que $X$ é da classe $i$ é dada por (Swain \& Davis 1978):

$$
L_{x}(i)=\sum_{j=1}^{m} \lambda(i / j) p(j / X)=\lambda(i / 1) p(1 / X)+\lambda(i / 2) p(2 / X)+\ldots+\lambda(i / m) p(m / X)
$$

sendo $p(j \mid X)$ a probabilidade a posteriori de atribuir a classe $j$ ao pixel $X$. Esta fórmula é uma esperança matemática da perda, por isso o termo "perda média". E a estratégia é selecionar a classe $i$ em que os valores de $p(j \mid X)$ sejam mínimos, quando $j \neq i$. Esta expressão gera várias funções discriminantes, uma para cada classe.

O pixel $X$ assumirá a classe que obtenha o valor mínimo para sua função, que é a estratégia de minimização da perda média. O princípio de tomada de decisão do algoritmo de máxima verosimilhança é concluído aqui, e os passos seguintes correspondem a simplificações da expressão (1).

Como não é possivel obter diretamente as probabilidades a posteriori, $p(j \mid X)$, a partir dos dados de treinamento, é preciso recorrer ao teorema de Bayes, $p(j \mid X)=p(X \mid j) p(j) / p(X)$, e substituir em $L_{x}(i)$, conforme expressão a seguir:

$$
L_{x}(i)=\sum_{j=1}^{m} \lambda(i / j) p(X / j) p(j) / p(X)
$$

$p(X \mid j)$ corresponde à informação da evidência de um pixel em relação a classe amostrada de treinamento e é obtida por meio da função multinormal com os dados do vetor de média da classe $j$ e sua matriz de variância-covariância.

Swain e Davis (1978) descrevem três regras a serem aplicadas em (2) com a finalidade de simplificar a expressão. São elas:

1)Minimizar uma função é análogo a maximizar o negativo da mesma função;

2)Assumir os seguintes valores para a função de perdas, $\lambda(i \mid j): 0$, quando a classificação for correta $(i=j)$; e 1, quando a classificação for incorreta; e

3)Adotar funções monotônicas das funções discriminantes geradas pela equação (2).

Aplicando a regra 1, tem-se que $g_{i}(X)=-L_{x}(i)$, seguindo a notação em Swain e Davis (1978). $X$ agora irá pertencer àquela classe $i$ em que $g_{i}(X)$ é máximo, ou seja, a classe que minimiza a perda média.

Aplicando a regra 2, tem-se que: 


$$
g_{i}(X)=-\sum_{j=1}^{m} \lambda(i / j) p(X / j) p(j) / p(X)=-1 / p(X) \sum_{\substack{j=1 \\ j \neq i}}^{m} p(X / j) p(j)
$$

porque $\lambda(i \mid j)=0$ somente quando $i=j$, e $p(X)$ é uma constante, um valor fixo determinado pela amostra de treinamento.

Aplicando a regra 3, elimina-se o termo constante sem alterar a regra de decisão, resultando na expressão:

$$
g_{i}(X)=-\sum_{\substack{j=1 \\ j \neq i}}^{m} p(X / j) p(j)
$$

A expressão (3) ainda exige o cálculo com probabilidades de todas as classes, com exceção de $j=i$, para cada função discriminante. Para resolver esta complexidade da fórmula, utiliza-se a expressão de $p(X)=\Sigma p(X, j)=\Sigma p(X \mid j) p(j)$, pois $p(X \mid j)=p(X, j) / p(j)$. Pode-se escrever que

$$
p(X)=p(X / i) p(i)+\sum_{\substack{j=1 \\ j \neq i}}^{m} p(X / j) p(j)
$$

e isolando o somatório na expressão (5) e substituindo em (4), tem-se que

$$
g_{i}(X)=-p(X)+p(X / i) p(i)
$$
pressão

Aplicando novamente a regra 3 da função monotônica, chega-se finalmente à ex-

$$
g_{i}(X)=p(X / i) p(i)
$$

que reúne o conjunto de funções discriminantes, uma para cada classe, sendo a norma de otimização de Bayes descrita por: $X \in i$ se e somente se: $p(X \mid i) p(i) \geq p(X \mid j) p(j)$, para todo $j=1$, $2, \ldots, \mathrm{m}$.

Como o classificador de máxima verosimilhança é paramétrico, sendo as distribuições de pixels das classes, assumidas como funções densidade multinormais, substitui-se a função

$$
p(X / i)=\frac{1}{2 \pi^{n / 2}\left|\sum_{i}\right|^{1 / 2}} \exp \left[-1 / 2\left(X-U_{i}\right)^{T} \sum_{i}^{-1}\left(X-U_{i}\right)\right]
$$

em (6), e após a logaritmização, chega-se à fórmula final do algoritmo de máxima verosimilhança Gaussiana, eliminando-se o termo constante, conforme expressão (8)

$$
g_{i}(X)=\ln p(i)-(1 / 2) \ln \left|\sum_{i}\right|-(1 / 2)\left(X-U_{i}\right)^{T} \sum_{i}^{-1}\left(X-U_{i}\right)
$$

Este classificador permite ainda que se estabeleça um limiar probabilístico de pixels classificados. Pode-se estabelecer por exemplo, que se deseje classificar somente $95 \%$ dos pixels, correspondente a área sob a superfície de resposta de cada classe representada no espaço n-dimensional formado pelas bandas espectrais. Isto significa a não classificação de $5 \%$ de pixels que estariam margeando estas superfícies, mais afastados portanto das médias das classes. Estes pixels, devido à baixa freqüência, relativa à classe, apresentam geralmente um alto grau de incerteza, ou seja, um alto grau de mistura com outras classes, aumentando a chance de classificação incorreta.

A forma de se estabelecer este limiar não é pela distribuição normal, pois na expressão final a função de probabilidade $p(X \mid i)$ foi degenerada. Isto é feito com o termo quadrático, assumindo que este segue distribuição Chi-quadrado. Assim, utiliza-se valores tabelados ao 
Quadro 2: Matrizes de Variância-Covariância, suas Inversas, Determinantes e Vetores de Média para as Classes LAGOA e FLORESTA. Matrices of variance-covariance, its inverse ones, its determinants and its average vectors for the classes lagoon and Forest.

\begin{tabular}{|c|c|c|c|c|c|}
\hline \multirow[t]{2}{*}{ LAGOA } & \multirow{2}{*}{$\begin{array}{l}\text { MATRIZ } \\
\text { B'j }\end{array}$} & \multicolumn{2}{|c|}{ VARIÂNCIIA-CÓVARIÁNCIIA } & \multirow[t]{2}{*}{ DETERMINANTE } & \multirow[t]{2}{*}{ VETÓR DE MÉDIAS } \\
\hline & & B4 & 85 & & \\
\hline $\mathrm{B} \hat{3}$ & $0.6<12503$ & ป.03ิ5 ว625 & J1425ió13 & \multirow[t]{8}{*}{$0.3009<53$} & \multirow{8}{*}{$\begin{array}{l}12.4375 \\
5.5625 \\
1.466815\end{array}$} \\
\hline 34 & 0.03515625 & 0.36306452 & -i) 10742158 & & \\
\hline \multirow[t]{3}{*}{85} & $0.1<25 / 813$ & $0.10142^{\prime} 86$ & 1286431452 & & \\
\hline & MATRIZ & INVERSA & & & \\
\hline & $\mathrm{B} 3$ & B1 & 85 & & \\
\hline B3 & 1.60278543 & -0.69972386 & 016893143 & & \\
\hline 84 & -0.09972380 & 2.6791836 & $02 \times 2 \times 9375$ & & \\
\hline$B 5$ & 0.16893413 & $0.212 \times 9375$ & $08 \times 196882$ & & \\
\hline \multirow[t]{2}{*}{ FLORESTA } & MATRLZ & VARIANCIA & COVARIÅNCIA & DETERMINANIE & \multirow[t]{2}{*}{ VETOR DE MÉDIAS } \\
\hline & B33 & L34 & 35 & & \\
\hline B.3 & $1.1378 \times 5 \cdot 3$ & 5.11183673 & 1 56897959 & \multirow[t]{8}{*}{556.09974} & \multirow{8}{*}{$\begin{array}{l}16.4571429 \\
54.9478571 \\
45.9428571\end{array}$} \\
\hline B4 & $5.111 \hat{3} 3673$ & 61.2907563 & 11. ถิ4453 13 & & \\
\hline \multirow[t]{3}{*}{ B5 } & 1.568897959 & 11. 6445313 & 15.4672269 & & \\
\hline & MATRIZ & \multicolumn{2}{|l|}{ INVERSA } & & \\
\hline & B3 & \multicolumn{2}{|r|}{ 85 } & & \\
\hline 83 & 1.46009427 & -0.10032554 & -006588587 & & \\
\hline 84 & -0.109332554 & 0.0272232 & $-1) 00040231$ & & \\
\hline 85 & .0065835667 & -0.60040201 & 0.0704152 & & \\
\hline
\end{tabular}

nível de probabilidade desejado (\% de pixels a classificar). Se o resultado dos termos quadráticos para um determinado pixel $(X)$, nas funções discriminantes de todas as classes ultrapassar o valor tabelado, este pixel não é classificado.

Os dados para a expressão (8), que são: as matrizes de variância-covariância, $\sum_{i}$, seus determinantes, suas inversas, e vetores de média, $U_{i}$, são obtidos das amostras de treinamento "Lagoa" e "Floresta", que foram extraídas da imagem, conforme quadro 2:

Os valores calculados pela expressão (8), considerando probabilidades a priori, $p(i)$ iguais, para os valores de brilho de quatro pixels da subcena $20 \times 20$ pixels são apresentados no quadro 3 :

Quadro 3: Coordenadas [Linha, Coluna] dos pixels, seus Valores de Brilho (VB) nas Bandas 3, 4 e 5, valores das funções discriminantes e atribuição das classes aos respectivos pixels, pela regra de decisão. Coordinates [line, column] of the pixels, its brightness values (BV) in the 3,4 and 5 bands, values of the discriminating functions and attribution of the classes to the respective pixels, by the rule of decision.

\begin{tabular}{|c|c|c|c|c|c|c|}
\hline \multirow[b]{2}{*}{ Pixel } & \multicolumn{3}{|c|}{ VR } & \multirow[b]{2}{*}{$\dot{y} A_{i j \cdot u t}(X)$} & \multirow[b]{2}{*}{ 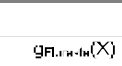 } & \multirow[b]{2}{*}{ Clysse } \\
\hline & 33 & B4 & B5 & & & \\
\hline$[0,0]$ & 13 & $\mathrm{E}$ & 2 & -3.66028445 & -71.4712057 & -agoa \\
\hline$[4,0]$ & 14 & 13 & 23 & -305.935086 & -28.1218161 & Floresta \\
\hline$[4,5]$ & 15 & 73 & 29 & -832.802011 & -18.0497983 & Floresta \\
\hline$[15,15]$ & 17 & 52 & 39 & -3858.08246 & -5.61139175 & Floresta \\
\hline
\end{tabular}




\section{O Classificador não Paramétrico Supervisionado de Skidmore/Turner (NPSKID)}

Este classificador também utiliza o teorema de Bayes para desenvolver sua regra de decisão, conforme expressão:

$$
P(i \mid X)=\frac{p(i \cap X)}{p(X)}=\frac{p(X i) P(i)}{\sum_{j=}^{k} p(X \mid j) P(j)}
$$

O denominador, $p(X)$, que corresponde à soma de interseções do vetor $X$ com todas as classes, é um valor constante para o pixel $X$, e, em sua forma original, não é descartado como no MAXVER. Mas a principal diferença em relação ao MAXVER, está no cálculo de $p(X \mid j)$. Os dados de treinamento para este classificador constituem-se em conjuntos discretos de vetores de pixels, comuns a cada classe, e não mais um espaço contínuo determinado pela função multinormal. $O$ que irá determinar se o pixel $X$ pertence à classe $j$, será o valor máximo da sua freqüência de ocorrência nesta classe, ponderada pelo número de pixels amostrados na classe $j$, em relação às outras classes. Isto é demostrado, transformando-se as probabilidades para freqüências, possíveis de extrair da amostra de treinamento.

Como o estimador para $P(X \mid i)$ é:

$$
F(X \mid i)=\frac{p(X \cap i)}{p(i)}=\frac{F(X \cap i) / \boldsymbol{F}}{F_{i} / F}=\begin{gathered}
F(X \cap i) \\
F
\end{gathered}
$$

E substituindo-se (10) em (9), a expressão apresentada em Skidmore e Turner (1988) após errata apresentada em Lowell (1989), e correção da notação em Gong e Dunlop (1991), é:

$$
P(i \mid X)-\frac{\left(F / F_{i}\right) F(i \cap X) P(i)}{\sum_{j=1}^{k}(F / F) F(j \cap X) P(j)}-\frac{\left(1 / F_{i}\right) F(i \cap X) P(i)}{\sum_{j=1}^{k}\left(1 / F_{j}\right) F(j \cap X) P(j)}
$$

eliminando-se o $F$, total das amostras de treinamento, que é comum ao numerador e ao denominador.

Sendo:

$P(i \mid X)=$ probabilidade empírica, ou a posteriori. É a probabilidade de se atribuir a classe $i$ ao vetor de pixel $X$;

$F=$ total de pixels da amostra de treinamento;

$F_{i} ; F_{j}=$ frequência de pixels amostrados para classe $i$ e $j$ respectivamente;

$F(j \cap X)=$ frequência de ocorrência do vetor de pixel $X$ com a classe j;

$P(i) ; P(j)=$ probabilidades a priori das classes $i$ e $j$ respectivamente;

$k=$ número de classes.

$F / F_{i} ; F_{j}=$ normalização para eliminar a influência da diferença de áreas amostradas entre classes nos resultados.

A expressão (11), como descrito em Gong e Dunlop (1991), pode ainda ser simplificada, eliminando-se o denominador, que para o pixel $X$, é constante a todas as classes, podendo ser rescrita como:

$$
P(i \mid X)=\left(1 / F_{i}\right) F(i \cap X) P(i)
$$

A classe que obtiver a maior pertinência em (12), é assumida ao pixel $X$. O algoritmo passa então para o próximo pixel e repete o processo até varrer toda a imagem. Se o vetor $X$ não ocorrer em nenhuma classe amostrada, este pixel não será classificado. 
O processo exige um grande esforço amostral, pois em dados de sensoriamento remoto é grande a variabilidade tonal de uma determinada feição ou classe informacional, devido a diversos fatores, como resolução espacial (mais de uma classe dentro do IFOV), topografia do terreno (interferência do sombreamento no IFOV, em diferentes intensidades), e principalmente ruídos, causados pelos próprios sensores durante o imageamento.

Com os dados do quadro 1, executou-se a regra de decisão de Skidmore/Turner, (11), e sua simplificação, descrita em Gong e Dunlop (1991), (12), para os quatro pixels, considerando-se probabilidades a priori iguais. Os resultados são apresentados no quadro 4 .

Quadro 4: Coordenadas [Linha, Coluna] dos pixels, seus Valores de Brilho (VB) nas Bandas 3, 4 e 5, valores das funções discriminantes de Skidmore/Turner e de Gong e Dunlop, e atribuição das classes aos respectivos pixels, pela regra de decisão. Coordinates [line, column] of the pixels, its brightness values (BV) in the 3, 4 and 5 bands, its values of the expressions of Skidmore/Turner and of Gong and Dunlop and attribution of the classes to the respective pixels, for the rule of decision.

\begin{tabular}{c|ccc|rcccc}
\hline & \multicolumn{3}{|c|}{ VB } & \multicolumn{3}{c}{ Skidmore /Turner } & \multicolumn{3}{c}{ Gong e /Dunlop } \\
\hline PIxal & B3 & B4 & B5 & P(Lagoa) & P(Floresta) & P(Lagoa) & P(Floresta) & Classe \\
\hline$[0,0]$ & 13 & 6 & 2 & 1 & 0 & 0,0625 & 0 & Lagoa \\
{$[4,0]$} & 14 & 13 & 23 & 0 & 0 & 0 & 0 & - \\
{$[4,5]$} & 15 & 23 & 29 & 0 & 0 & 0 & 0 & - \\
{$[15,15]$} & 13 & 52 & 39 & 0 & 0 & 0 & 0 & - \\
\hline
\end{tabular}

$$
\begin{array}{ll}
P(\text { Lagoa } \mid[0,0])=\frac{(1 / 32) * 2}{1 / 32 * 2+1 / 35 * 0}=1 & P(\text { Floresta } \mid[0,0])=\frac{(1 / 35) * 0}{1 / 32 * 2-1 / 35 * 0}=0 \\
P(\operatorname{Lagroa} \mid[4,0])=\frac{(1 / 32) * 0}{1 / 32 * 0+1 / 35 * 0}=0 & P(\text { Floresla } \mid[1,0])=\frac{(1 / 35) * 0}{1 / 32 * 0-1 / 35 * 0}=0 \\
P(\operatorname{Lagoa} \mid[4,5])-\frac{(1 / 32) * 0}{1 / 32 * 0-1 / 35 * 0}-0 & P(\text { Floresta } \mid[4,5])-\frac{(1 / 35) * 0}{1 / 32 * 0-1 / 35 * 0}-0 \\
P(\operatorname{Lagoa} \mid[15,15])-\frac{(1 / 32) * 0}{1 / 32 * 0+1 / 35 * 0}-0 & P(\text { Floresta } \mid[15,15]) \frac{(1 / 35) * 0}{1 / 32 * 0+1 / 35 * 0}=0
\end{array}
$$

Os pixels $[4,0],[4,5]$ e $[15,15]$ não foram classificados porque não ocorreram nas amostras de treinamento (quadro 1). Comparando seus valores de brilho, com a amplitude dos valores de brilho das classes de treinamento no quadro 1, pode-se inferir que os pixels $[4,0]$ e $[4,5]$ contém informação de mistura entre as duas classes, estando o [4,5] mais similar à FLORESTA. Já o pixel $[15,15]$ contém informação típica de Floresta, apesar de seus valores de brilho 52, na Banda 4, e 39, na Banda 5 não ocorrerem na amostra.

Esta restrição do classificador não paramétrico de Skidmore/Turner não ocorre para o algoritmo de máxima verosimilhança, pois a baixa representatividade da amostra é corrigida pelo ajuste da multinormal, formando um espaço contínuo para a classe. Desta forma, não ocorrem interrupções nos histogramas.

\section{O Classificador não Paramétrico Supervisionado NPVIC}

Com a finalidade de reduzir o efeito restritivo do classificador não paramétrico de Skidmore/ Turner foi desenvolvido o NPVIC, um algoritmo supervisionado não paramétrico, por pixel, que teve como princípio flexibilizar a rígida associação entre os valores de brilho do vetor de pixel.

Este algoritmo, desenvolvido a partir da probabilidade condicional, $P(i \mid X)$, considera os valores de brilho independentes por banda, e atribui ao pixel a classe que integra o valor máximo de interseções por bandas, ponderadas pelos tamanhos das classes.

As bandas de uma imagem são mutuamente exclusivas. Ou seja, um valor de brilho 
$\left(x_{i}\right)$ de um pixel qualquer na Banda 3 não pertence à Banda 4 ou 5.

A expressão da probabilidade condicional é dada por:

$$
P(i \mid X)=\frac{p(i \cap X)}{p(X)}=\frac{p(i \cap X)}{\sum_{i=1}^{k} p(j \cap X)}
$$

sendo $X$, o vetor de pixels com três bandas. Para demonstração considera-se duas classes. A dedução para $P(i \mid X)$, aplicando as propriedades de probabilidade condicional e distributiva, é dada a seguir.

$$
P(i \mid X)=P\left(i \cup i_{2} \cup i_{3}, x_{1} \cup x_{3} \cup x_{3}\right)=\begin{gathered}
P\left[\left(i_{1} \cup i_{2} \cup i_{3}\right) \cap\left(x_{1} \cup x_{2} \cup x_{3}\right)\right] \\
P\left(x_{1} \cup x_{2} \cup x_{3}\right)
\end{gathered}
$$

sendo:

$i_{1}, i_{2}, i_{3}$, a classe $i$ considerando seus valores de brilho nas Bandas 1, 2 e 3 respectivamente; analogamente para $x_{1}, x_{2}, x_{3}$.

$P(i \mid X)=\frac{I\left[\left(i \cap x_{1}\right) \cup\left(i \cap x_{2}\right) \cup\left(i_{1} \cap x_{3}\right) \cup\left(i_{2} \cap x_{1}\right) \cup\left(i, r x_{2}\right) \cup\left(i_{2} \cap x_{2}\right) \cup\left(i_{3} \cap x\right) \cup\left(i_{2}, \cap x_{2}\right) \cup\left(i_{2}, \cap x_{3}\right)\right]}{P\left(x_{1} \cup x_{2} \cup x_{3}\right)}$

Como as Bandas são mutuamente exclusivas, qualquer interseção que relacione valores de brilho entre Bandas é zero. Com isso, a fórmula se reduz para:

$P(i \mid X)-\frac{P\left[\left(i_{1} \curvearrowright x_{2}\right) \cup\left(i_{2} \curvearrowright x_{2}\right) \cup\left(i_{3} \cap x_{3}\right\}\right.}{P\left(x \cup x_{2} \cup x_{1}\right)}-\frac{\left.P\left(i_{1} \cap x_{1}\right)-\left(i_{2} \cap x_{2}\right)-\left(i_{3} \curvearrowright x_{3}\right)\right]}{P\left(x_{1}+x_{2}+x_{3}\right)}-\frac{p\left(i_{1} \rho x_{2}\right)+p\left(i_{2} \curvearrowright x_{2}\right)+p\left(i_{3} \cap x_{3}\right)}{p(x)+p\left(x_{2}\right)+p\left(x_{1}\right)}$

Transformando esta expressão para frequência, tem-se que

$$
P(i \mid X)=\frac{\frac{F\left(i_{1} \cap x_{1}\right)}{F\left(i_{1}\right)+F\left(x_{1}\right)-F\left(i_{1} \cap x_{1}\right)} \frac{F\left(i_{2} \cap x_{2}\right)}{F\left(i_{2}\right)+F\left(x_{2}\right)-F\left(i_{2} \cap x_{2}\right)}+\frac{F\left(i_{3} \cap x_{3}\right)}{F\left(i_{3}\right)+F\left(x_{3}\right)-F\left(i_{3} \cap x_{3}\right)}}{\frac{F(x)+F\left(x_{2}\right)+F\left(x_{3}\right)}{F}}
$$

$F\left(i_{n}\right)$ é o tamanho da amostra para a classe $i$ na Banda $n$, o número de pixels amostrados, que para LAGOA, foi 32. Este valor é constante para as $\mathrm{n}$ bandas, e pode ser colocado em evidência na forma de $F(i)$, o fator de ponderação pelo tamanho da classe. $F\left(x_{n}\right)$ é a freqüência do valor de brilho $(x)$ na banda $n$. Estes valores são fixos, independentes das classes, e podem ser eliminados da expressão, assim como a subtração dos termos $F\left(i_{n} \cap x_{n}\right)$, presentes apenas para representação teórica de $F\left(i_{n} \cup x_{n}\right)$, mas sem nenhum efeito na regra decisória entre classes. $O$ mesmo pode-se dizer para o total de pixels da amostra de treinamento, $F$, presente no denominador.

A expressão é assim simplificada para:

$$
g_{(i \mid x ;}=\frac{1}{F(i)}\left[F\left(i_{1} \cap x_{1}\right)+F\left(i_{2} \cap x_{2}\right)+F\left(i_{3} \cap x_{3}\right)\right]
$$

O fator de normalização é inerente à dedução e não necessita ser adicionado como na expressão em Skidmore e Turner (1988), e as probabilidades a priori desaparecem. 
COSTA, T. C. C. et al. Classificação do uso/cobertura da terra por meio de...

Quadro 5 - Histogramas de frequência para as Classes "LAGOA" e "FLORESTA" para as Bandas 3,4 e 5 do Landsat5-TM. Frequency histograms for the classes lagoon and forest for the 3,4 and 5 Landsat TM bands.

\begin{tabular}{|c|c|c|c|c|c|}
\hline \multicolumn{6}{|c|}{ LAGOA } \\
\hline \multicolumn{2}{|r|}{ B.3 } & \multicolumn{2}{|r|}{$\mathrm{B} 4$} & \multicolumn{2}{|r|}{$\mathrm{B} 5$} \\
\hline VB & Frequéncia & VB & Frequéncia & VB & Frequeencia \\
\hline 11 & 4 & 4 & 2 & 0 & 8 \\
\hline 12 & 12 & 5 & 10 & 1 & 7 \\
\hline 13 & 14 & 6 & 20 & 2 & 13 \\
\hline \multirow[t]{2}{*}{14} & 2 & & & 3 & 2 \\
\hline & & & & 4 & 2 \\
\hline Total & 32 & & 32 & & 32 \\
\hline \multicolumn{6}{|c|}{ FLORESTA } \\
\hline \multicolumn{2}{|r|}{$\mathrm{B} 3$} & \multicolumn{2}{|c|}{$\mathrm{B} 4$} & \multicolumn{2}{|r|}{ B5 } \\
\hline VB & Frequencia & VB & Frequência & VB & Frequentia \\
\hline 14 & 1 & 25 & 1 & 35 & 1 \\
\hline 15 & 6 & 38 & 1 & 37 & 1 \\
\hline 16 & 9 & 39 & 1 & 40 & 2 \\
\hline 17 & 15 & 47 & 1 & 41 & 1 \\
\hline 18 & 3 & 48 & 1 & 42 & 2 \\
\hline \multirow[t]{14}{*}{19} & 1 & 49 & 1 & 43 & 2 \\
\hline & & 51 & 1 & 45 & 3 \\
\hline & & 52 & 1 & 46 & 5 \\
\hline & & 53 & 1 & 47 & 2 \\
\hline & & 54 & 2 & 48 & 6 \\
\hline & & 55 & 1 & 49 & 3 \\
\hline & & 56 & 2 & 50 & 6 \\
\hline & & 57 & 2 & 51 & 1 \\
\hline & & 58 & 6 & & \\
\hline & & 59 & 5 & & \\
\hline & & 60 & 4 & & \\
\hline & & 61 & 1 & & \\
\hline & & 62 & 2 & & \\
\hline & & 64 & 1 & & \\
\hline Tolal & 35 & & 35 & & 35 \\
\hline
\end{tabular}

Quadro 6 - Coordenadas [Linha, Coluna] dos pixels, seus Valores de Brilho (VB) nas Bandas 3, 4 e 5, valores das funções discriminantes do NPVIC, e atribuição das classes aos respectivos pixels, pela regra de decisão. Coordinates [line, column] of the pixels, its brightness values (BV) in the 3, 4 and 5 bands, its values of the expression of NPVIC and attribution of the classes to the respective pixels, for the rule of decision.

\begin{tabular}{c|rcc|rrc}
\hline & \multicolumn{3}{|c|}{ VB } & & & \\
\hline Pixel & B3 & B4 & B5 & g(Lagoa) & g(Floresta) & Classe \\
\hline$[0,0]$ & 13 & 6 & 2 & 1,16875 & 0 & Lagaa \\
{$[4,0]$} & 14 & 13 & 23 & 0,0625 & 0,0286 & Lagaa \\
{$[4,5]$} & 15 & 23 & 29 & 0 & 0,1714 & Floresta \\
{$[15,15]$} & 17 & 52 & 39 & 0 & 0,4571 & Floresta \\
\hline
\end{tabular}

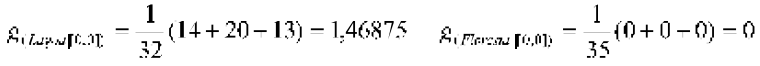

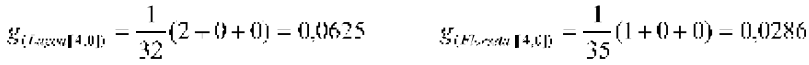

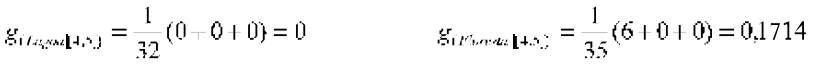

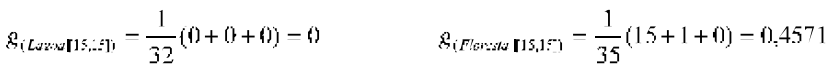


Para o cálculo da expressão 14 com os dados do quadro 1, é preciso transformar os vetores de pixels em freqüências de valores de brilho por banda, conforme quadro 5 .

Com os dados do quadro 5 , executa-se a regra de decisão do NPVIC, (14) para os quatro pixels, com resultados no quadro 6 .

Nota-se que ocorreu divergência na classificação do pixel $[4,0]$ em relação ao MAXVER, que o classificou como FLORESTA.

\section{O Classificador não Paramétrico de Skidmore/Turner "Melhorado" por Dymond (1993), NPSKIDYM}

Dymond fez a seguinte proposta de modificação no algoritmo de Skidmore/Turner: A ponderação da frequêencia de ocorrência do pixel na classe $j, F(X \cap j)$, não será feita pelo tamanho da classe $j, F_{j}$, mas pela freqüência média da classe, excluindo-se os valores que não ocorrem (freqüência zero). A freqüência média da classe é o tamanho da classe, $F_{j}$, dividido pelo número de valores distintos na classe, $N_{j}$. A inclusão desta normalização na regra de decisão aumenta a chance para classes de maior variabilidade, pois $N_{j}$ é proporcional ao desvio-padrão do histograma n-dimensional (Dymond 1993). A nova regra de decisão é assim descrita

$$
g(i \mid X)=\frac{N_{i}}{F_{i}} F(i \cap X) P(i) \text { sendo }
$$
classe $i$.

$N_{i}=$ soma de valores diferentes de zero que ocorrem no espaço n-dimensional da

\section{O Classificador NPVIC com a Estratégia de Dymond (1993), NPVICDYM}

A normalização pela freqüência da classe também foi implementada no NPVIC, resultando na expressão:

$$
g(i \mid X)=\frac{1}{F_{i}}\left[N_{i 1} * F\left(i_{1} \frown x_{1}\right)-N_{i 2} * F\left(i_{2} \cap x_{2}\right)+N_{i 3} * F\left(i_{3} \cap x_{3}\right)\right]
$$

sendo $N_{i n}=$ número de valores diferentes de zero na classe $i$ para a banda $n$.

Executou-se, portanto para exemplo, a regra de decisão de Skidmore/Turner, simplificada por Gong e Dunlop e "melhorada" por Dymond (15), e o NPVIC com a normalização proposta por Dymond (1993), (16), para os quatro pixels, considerando-se probabilidades a priori iguais. Os resultados são apresentados no quadro 7 .

\section{MATERIAIS E MÉTODOS}

\section{Área de Estudo}

A área está situada na microrregião de Viçosa-MG, com predominância neste Município (figura 1). Seu relevo é ondulado com $43 \%$ da área com declividades entre 0 a $10^{\circ}, 56 \%$ entre 10 e $30^{\circ}$ e $1 \%$ acima de $30^{\circ}$. A tipologia vegetal é a Floresta Estacional Semidecidual, sob domínio da Mata Atlântica.

Foi utilizada uma subcena de $15 \times 15 \mathrm{~km}$ (22.500 ha), extraída da imagem Landsat5TM, Órbita 217 Ponto 74, Quadrante Sul, de 10/10/94, composta de 3 bandas espectrais: TM3, TM4 e TM5. A correção geométrica da imagem foi feita com pontos de controle coletados em OrtofotoCartas da CEMIG, Escala 1:10.000, utilizando-se a função de mapeamento quadrática, com reamostragem pelo vizinho mais próximo, atingindo um RMS $<15$ metros.

A referência da área (Verdade de Campo) foi obtida com o apoio de 14 Fotos aéreas não convencionais, na Fscala aproximada de 1:15.000, tiradas com duas câmeras Hasseblad 
COSTA, T. C. C. et al. Classificação do uso/cobertura da terra por meio de...

Quadro 7 - Coordenadas [Linha, Coluna] dos pixels, seus Valores de Brilho (VB) nas Bandas 3, 4 e 5, valores das funções discriminantes de Skidmore/Turner e de Dymond, e atribuição das classes aos respectivos pixels, pela regra de decisão. Coordinates [line, column] of the pixels, its brightness values (BV) in the 3, 4 and 5 bands, its values of Skidmore/Turner's and of Dymond's discriminating functions and attribution of the classes to the respective pixels, by the rule of decision.

\begin{tabular}{|c|c|c|c|c|c|c|c|}
\hline \multirow[b]{2}{*}{ Pixel } & \multicolumn{2}{|r|}{ VB } & \multicolumn{2}{|c|}{$\begin{array}{r}\text { Skidmore/Tur "melhorado" } \\
\text { n por Dymond }\end{array}$} & \multicolumn{2}{|c|}{$\begin{array}{r}\text { NPVIC "melhorado" } \\
\text { por Dymond }\end{array}$} & \multirow[b]{2}{*}{ Classe } \\
\hline & B3 & B4 B5 & $P($ Lagoa $)$ & $\mathrm{P}$ (Floresta) & $\mathbf{P}$ (Lagoa) & $\mathrm{P}($ Floresta $)$ & \\
\hline$[0,0]$ & 13 & 6 & 0,75 & 0 & 5,656 & 0 & Lagoa \\
\hline$[4,0]$ & 14 & 1323 & 0 & 0 & 0,25 & 0.1714 & Lagna \\
\hline$[4,5]$ & 152 & 2329 & 0 & 0 & 0 & 1,0286 & Floresta \\
\hline$[15,15]$ & 17 & $52 \quad 39$ & 0 & 0 & 0 & 3,1143 & Floreste. \\
\hline
\end{tabular}

Cálculos com expressäo (15): Skidmore/Turner "melhorado" por Dymond

$$
\begin{aligned}
& P(\text { Lagoa } \| 0,0])=(12 / 32) * 2=0,75 \\
& P(\text { Lagoa } \mid[4,0])-(12 / 32) * 0-0 \\
& P(\text { Lagoa } \| 4,5])=(12 / 32) * 0=0 \\
& P(\text { Lagoa } \| 15,15])=(12 / 32) * 0=0
\end{aligned}
$$

$$
\begin{aligned}
& P(\text { Floresta } \| 0,0])=(38 / 35) * 0=0 \\
& P(\text { Flonesta } \| 4,0])-(38 / 35) * 0-0 \\
& P(\text { Floresta } \| 4,5])=(38 / 35) * 0=0 \\
& P(\text { Floresta } \| 15,15])=(38 / 35) * 0=0
\end{aligned}
$$

Cálculos com expressăo (16): NPVIC "melhorado" por Dymond

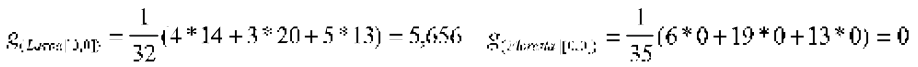

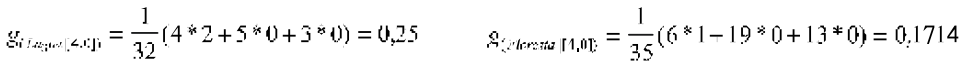

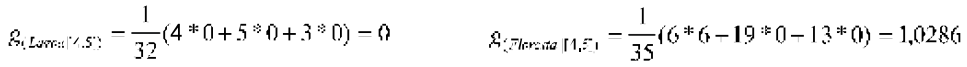

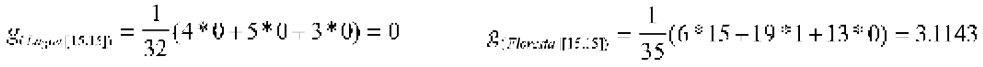

modelo 553 ELX, com objetivas Zeiss Distigon 4/50 mm T acopladas em um avião CESSNA 182-Skylane, do Depto de Solos da UFV. Na Figura 2 é apresentada a composição colorida 543 (RGB), com a cobertura das fotos aéreas e amostras de treinamento, e na Figura 3 a dispersão bidimensional das amostras de treinamento, tendo as bandas como eixo.

Foram definidas 11 classes de Cobertura/Uso da Terra (Quadro 8), interpretadas nas fotos aéreas não convencionais na escala 1:15.000, excluindo-se queimada (de baixa freqüência na imagem), fazendo-se a fusão entre pasto sujo e limpo, e capoeira e mata secundária, devido a pobre separabilidade entre estes pares de classes, medida pela Divergência Transformada. Na Figura 3 são apresentados os gráficos de dispersão das amostras de treinamento. Conforme recomendações em Swain e Davis (1978), e Jensen (1996) nenhuma amostra de treinamento ficou abaixo do limite de 30 pixels. As classes de treinamento estão descritas no quadro 8.

A amostra de referência foi constituída por uma grade sistemática de 163 pontos distantes de 28 pixels sobre a área das fotos, mais 146 pontos intermediários entre os pontos da grade (Total de 309 pontos). Esta amostra foi dimensionada pela fórmula da distribuição binomial: $n=\mathbb{Z} p q / E^{2}$, fixando-se os seguintes parâmetros: $p=0,76$, exatidão estimada por amostragem piloto (Costa 1999), $q=1-p ; Z \sim N(0,1)=1,96$ para $95 \%$ de confiança, e $E=0,05$, erro de amostragem, resultando em $n=280$, mais 29 pontos excedentes. $O$ quadro 8 mostra o número de pixels utilizados para treinamento e para referência. 


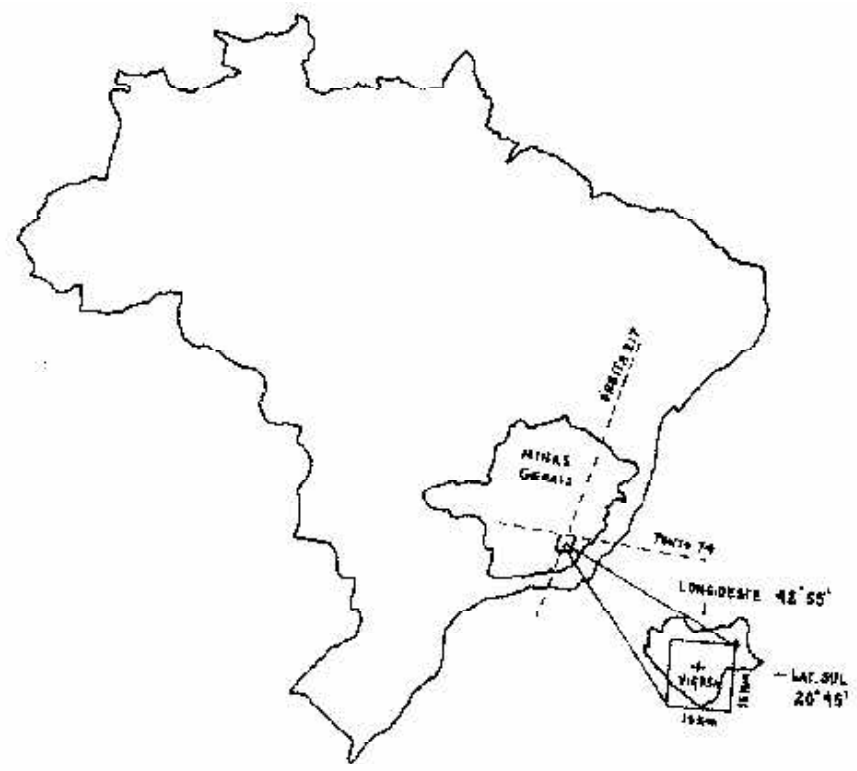

Figura 1 - Área de Estudo. Study of the Area.

O índice utilizado para avaliar os resultados das classificações foi a Exatidão Global (G). Para testar a diferença entre os índices de exatidão $\left(G_{1}\right.$ e $\left.G_{2}\right)$, o teste estatístico utilizado foi o de Z, conforme a expressão (Ma \& Redmond 1995):

$$
Z=\frac{G_{2}-G_{1}}{\sqrt{V\left(G_{2}\right)+V\left(G_{1}\right)}}
$$

sendo

$$
G=p=\frac{1}{n} \sum_{i=1}^{K} n_{i i} \text { e } \quad V(G)=\frac{p q}{n} .
$$

Para o cálculo de $\mathrm{G}$ não foi considerado o cruzamento de pontos de referência com áreas não classificadas da imagem, pois pixels não classificados não são erros de decisão, mas uma limitação dos algoritmos não paramétricos de Skidmore/Turner e NPVIC, avaliados pela \% de área não classificada.

\section{Classificadores}

O algoritmo Não Paramétrico de Skidmore/Turner, de Skidmore/Turner "melhorado" por Dymond, NPVIC, e NPVIC com normalização de Dymond foram programados em Pascal, aproveitando-se os módulos de coleta de amostras de treinamento do Sistema de Informações Geográficas IDRISI 2.0 para Windows (Vetorização Screen e MAKESIG). O algoritmo MAXVER utilizado é um módulo do mesmo sistema. 


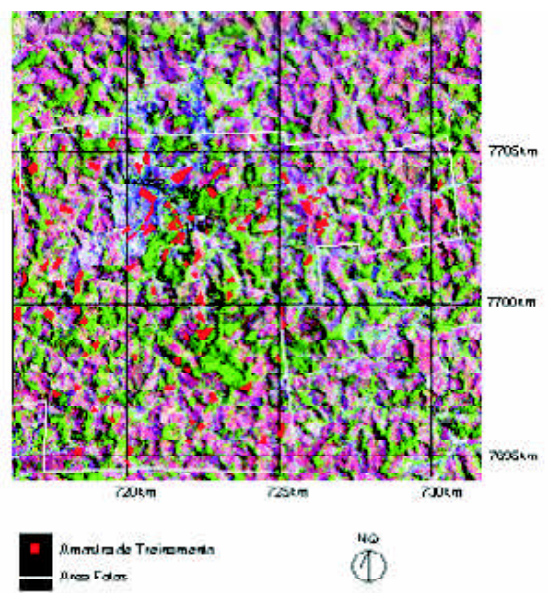

Figura 2 - Composição colorida 543 (RGB), a cobertura de fotos aéreas não convencionais e polígonos de treinamento. Color composition RGB 543, the non conventional cover arial photos and training samples.
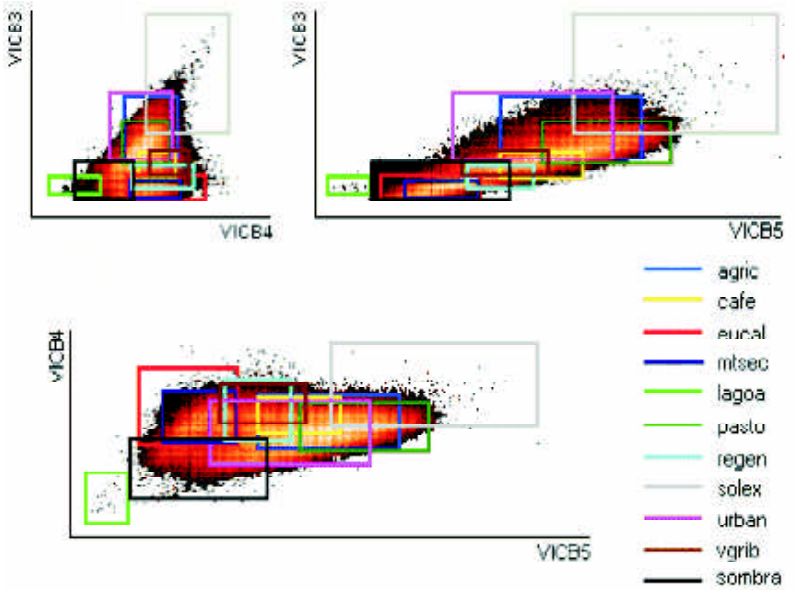

Figura 3 - Gráficos de dispersão das 11 classes obtidas pela amostra de treinamento, para as bandas $3 / 4 ; 3$ / 5 e 4/5. Scattergrams of the 11 classes obtained of the training samples of bands $3 / 4,3 / 5$ and $4 / 5$. 
COSTA, T. C. C. et al. Classificação do uso/cobertura da terra por meio de...

Quadro 8 - Descrição das Classes de Uso/Cobertura da Terra, e amostras de treinamento e referência por classe. The classes description about the land use/cover and training samples and reference for class.

\begin{tabular}{|c|c|c|}
\hline CLASSE & Pixels Treinam. & Pixels Refer. \\
\hline $\begin{array}{l}\text { 1 AGR|C: Area agricola, envolvendo solo arado, e culturas aruais em } \\
\text { diferertes estágios do dosonvolvimento }\end{array}$ & 233 & 27 \\
\hline 2.CAГE: Cafeicultura, com exposiçäo do solo & 363 & 11 \\
\hline 3.EUCAL: Eucalipla s em diéanles idades e lioos de nanejo & 24e & 11 \\
\hline 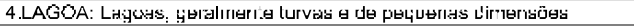 & 60 & 6 \\
\hline 5.MTSEC: Ma:a Serxındáriä err Estágyio Médio a Avar çado & 534 & 65 \\
\hline 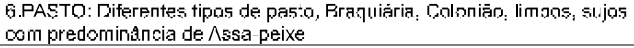 & 511 & 115 \\
\hline 7.REGEN: Nata Sccurdária em estagio inicial (regeneraçäo) & 71 & 21 \\
\hline 8.SOLEX: solo exoosto & 66 & 4 \\
\hline 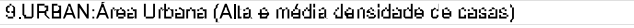 & 323 & 21 \\
\hline 10_VGRIB: vegetaçäo ribei inha (miaior a grarnirneas) & $5 \mathrm{~S}$ & 9 \\
\hline $\begin{array}{l}\text { 11.SOMBR: sombras, de grande ocarência devido a topogratia } \\
\text { acidentada da regiāo }\end{array}$ & $22 \mathrm{~B}$ & 19 \\
\hline TOTAL & 2996 & 309 \\
\hline
\end{tabular}

Foram ainda implementadas no classificador NPVIC as seguintes estratégias de classificação: (A) opção de aceitar a classificação de um pixel se ele apresentar interseção em 3 Bandas, 2 Bandas, ou classificá-lo sem restrição. A exigência da interseção em três Bandas reforça o conceito de assinatura espectral, que é o comportamento da feição ao longo do espectro. A ausência de restrição aumenta a possibilidade de classificação incorreta mas, por outro lado, aumenta a área classificada.

(B) Permite a classificação do pixel apenas se ele apresentar interseção máxima em 3 Bandas para a mesma classe, 2 Bandas, ou classificação sem restrição. Esta estratégia é mais restritiva do que a estratégia $(A)$.

\section{Seleção de Bandas}

A melhor combinação de dados para as respectivas classificações foi obtida em Costa (1999). Para testar as combinações de bandas e transformações de bandas, utilizou-se a divergência transformada, as transformações por Componentes Principais, Tasseled Cap e Discriminante Canônica, Índice Cramer's V, e Análise de Informação Mútua. O maior índice Kappa obtido foi para as Bandas 3, 4 e 5, sendo estas selecionadas para comparação entre os classificadores.

Os algoritmos foram aplicados sobre os dados originais (resolução radiométrica de 8 bits) das Bandas 3, 4, 5, e com dois fatores de compressão dos dados, reduzindo a resolução radiométrica para 7 bits; e para 6 bits, recomendados por Skidmore e Turner (1988) para aumentar a representatividade da amostra de treinamento.

\section{RESULTADOS E DISCUSSÃO}

Os resultados de exatidão global e percentual de área classificada na imagem estão apresentados no quadro 9. A compressão dos dados não solucionou o problema de área não classificada nos algoritmos NPSKID e NPSKIDYM. Dados comprimidos (menor número de bits) reduzem a área não classificada (\%ANC), mas também a exatidão. Embora estes classificadores tenham gerado imagens com exatidão global de $100 \%$ para os dados originais ( 8 bits), os mesmos classificaram apenas $3,10 \%$ da imagem, um pouco mais que as amostras de treinamento. Com a resolução de 7 bits, recomendado em seu artigo (Skidmore \& Turner 1988), a área não classificada atingiu $80,08 \%$, para uma exatidão global de $80,49 \%$. E mesmo com dados drasticamente comprimidos (6 bits), que têm a função de aumentar ainda mais a representatividade das amostras de treinamento, os algoritmos classificaram apenas $51,70 \%$ da imagem. 
COSTA, T. C. C. et al. Classificação do uso/cobertura da terra por meio de...

Quadro 9 - Exatidão Global (G) e \% de Áreas Não Classificadas (\%ANC) para a subcena de Viçosa classificada pelos algoritmos de Máxima Verosimilhança (MAXVER), Não Paramétrico de Skidmore/Turner (NPSKID); NPSKID com Estratégia de Dymond (NPSKIDYM); Não Paramétrico de Viçosa (NPVIC); NPVIC com Estratégia de Dymond (NPVICDYM) estratégias A e B (apenas para NPVIC), com 1, 2 e 3 interseções com as Bandas 3, 4, 5; sobre os dados originais ( 8 bits), com 7 bits; e 6 bits. Global accuracy (G) and \% of non-classified areas (\%ANC) for the subscene of Viçosa, classified by the algorithms of maximum likelihood (MAXLIKE), non-parametric of Skidmore/Turner (NPSKID); NPSKID with strategy of Dymond (NPSKIDYM); non-parametric of Viçosa (NPVIC); and NPVIC with strategy of Dymond (NPVICDYM), strategies $A$ and $B$ (just for NPVIC), with 1, 2 and 3 intersections with the Landsat TM 3, 4, 5 bands, on the original data, F1, and with two factors of compression of the data: FO, 5 , reducing the radiometric resolution to 7 bits; and FO, 25 reducing the resolution to 6 bits.

\begin{tabular}{|c|c|c|c|c|c|c|c|c|}
\hline \multicolumn{3}{|l|}{ ALG. CLAS. * } & \multicolumn{2}{|l|}{8 bits } & \multicolumn{2}{|l|}{7 bits } & \multicolumn{2}{|l|}{ 6. bits } \\
\hline & & N Band. Interses. & $\mathrm{G}$ & $(\% A N C)$ & $\mathrm{G}$ & $(\% A N C)$ & $\mathrm{G}$ & ( $\%$ ANC) \\
\hline MAXVERD $\%$ & & 3 & 76,37 & 0,00 & 73,79 & 0,00 & 74,11 & 0,00 \\
\hline MAXVER $1 \%$ & & 3 & 76,62 & 1,73 & 74,51 & 1,84 & 74,59 & 1,81 \\
\hline NPSKID & & 3 & 100 & 96.89 & 80,49 & 80,08 & 72,87 & 51,70 \\
\hline NPSKIDYM & & 3 & 100 & 96.89 & 80.49 & 80,08 & 73.40 & 51,70 \\
\hline \multirow[t]{5}{*}{ NPVIC } & A & 1 & 53,07 & 0,01 & 58,25 & 0,01 & 55,02 & 0,01 \\
\hline & & 2 & 62.16 & 16.01 & 64.39 & 9.60 & 64.89 & 9.78 \\
\hline & & 3 & 73.30 & 42,71 & 72.77 & 32,46 & 73,16 & 28,03 \\
\hline & $B$ & 2 & 63,38 & 61,63 & 68.87 & 61,10 & 72,73 & 61,73 \\
\hline & & 3 & 92,86 & 56,69 & $92: 86$ & 96,70 & 100 & 96,60 \\
\hline \multirow[t]{3}{*}{ NPVICDYM } & A & 1 & 55,02 & 0,01 & 56,63 & 0,01 & 64,40 & 0,01 \\
\hline & & 2 & 56,04 & 3.57 & 59,32 & 3,93 & 65,50 & 0,98 \\
\hline & & 3 & 64,78 & 26,12 & 65,00 & 20,99 & 70,57 & 13,78 \\
\hline
\end{tabular}

+Classificacāo com probabilidades a priori iguais para as classes.

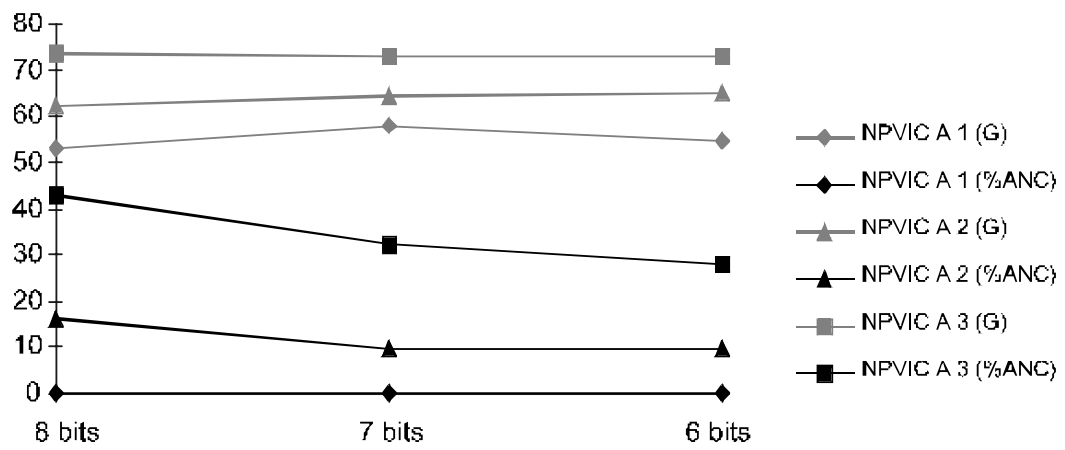

Compressão de dados

Figura 4 - Avaliação da Estratégia A do NPVIC para Exatidão Global (G) e \% de Área Não Classificada (\%ANC). Evaluation of the strategy A of NPVIC to global accuracy (G) and\% of non-classified area (\%ANC). 


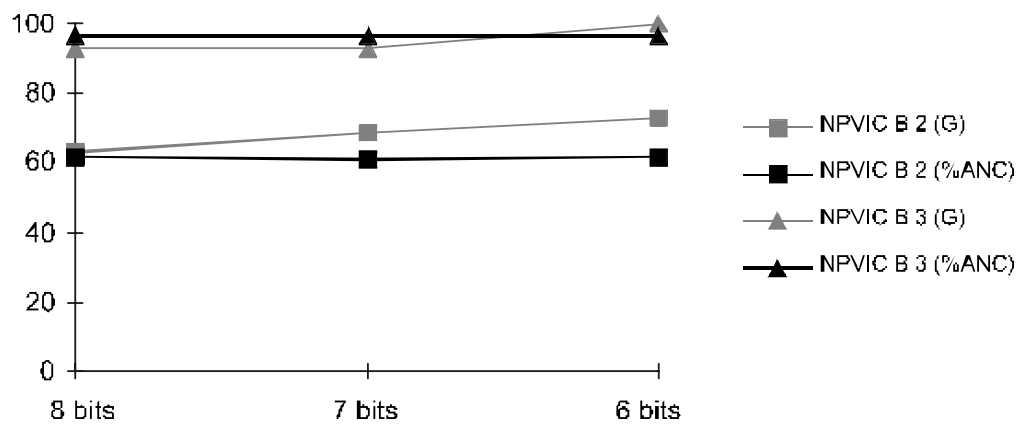

\section{Compressão de dados}

Figura 5 -Avaliação da Estratégia B do NPVIC para Exatidão Global (G) e \% de Área Não Classificada (\%ANC). Evaluation of the strategy $B$ of NPVIC to global accuracy (G) and \% of non-classified area (\%ANC).
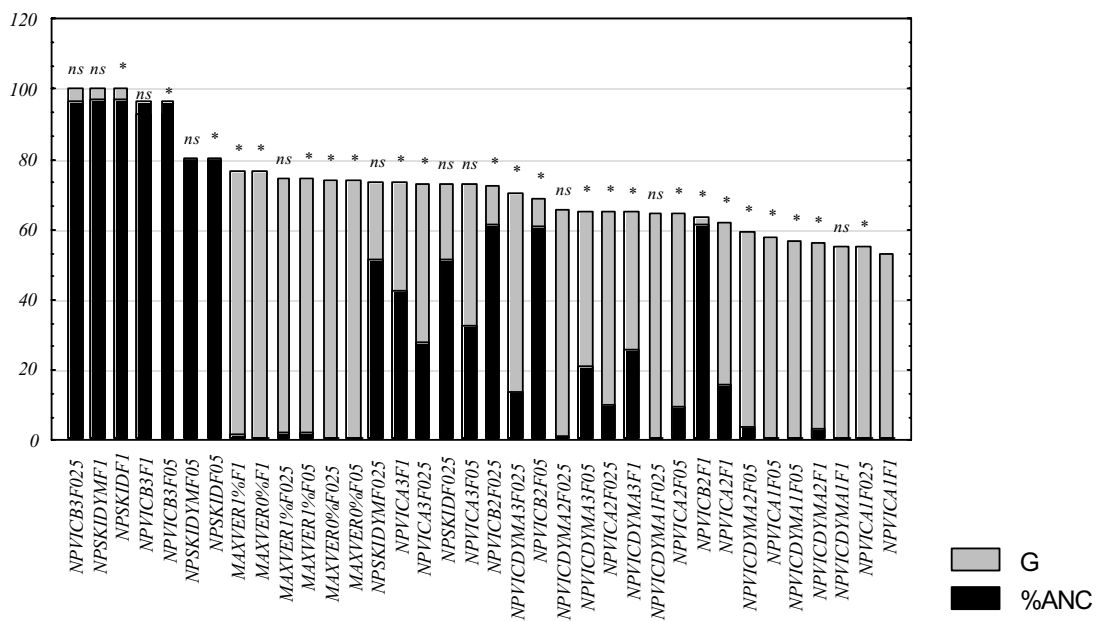

Figura 6 - Ranking dos algoritmos pela Exatidão Global (G), respectivas percentagens de Área Não Classificada (\%ANC) e teste Z seqüencial a $99 \%$ de probabilidade para G: * = signif. a $1 \%$; ns = não signif. a $1 \%$. Ranking of the algorithms by the global accuracy (G), respective percentage of non-classified area (\%ANC) and test $Z$ seqüencial at $99 \%$ of probability to G: * $=$ significant to $1 \%$ and ns $=$ non-significant at $1 \%$. 


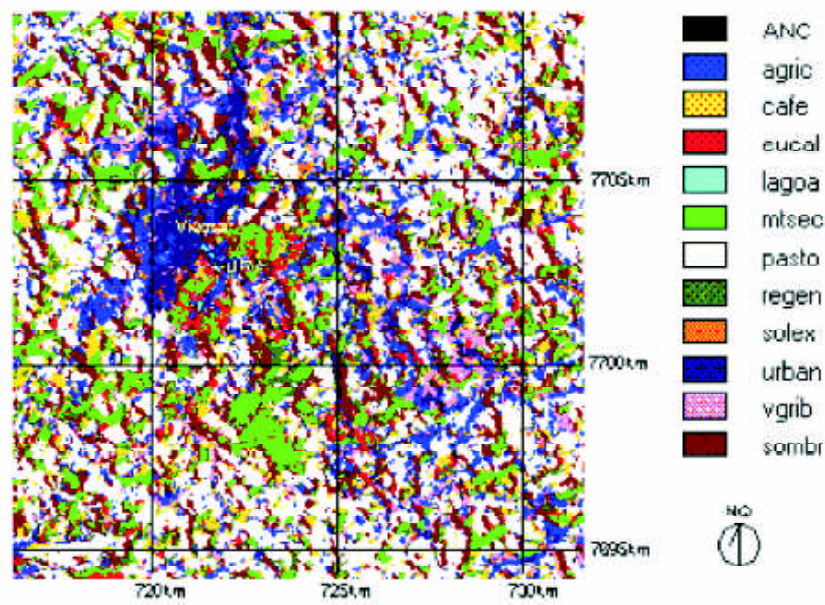

Figura 7 - MAXVER1\% pós-processada com filtro de moda/3×3. Grade UTM $(\mathrm{km})$ SAD69. MAXVER1\% pósprocessing with filter of the mode/3×3. Grade UTM $(\mathrm{km})$ SAD69.

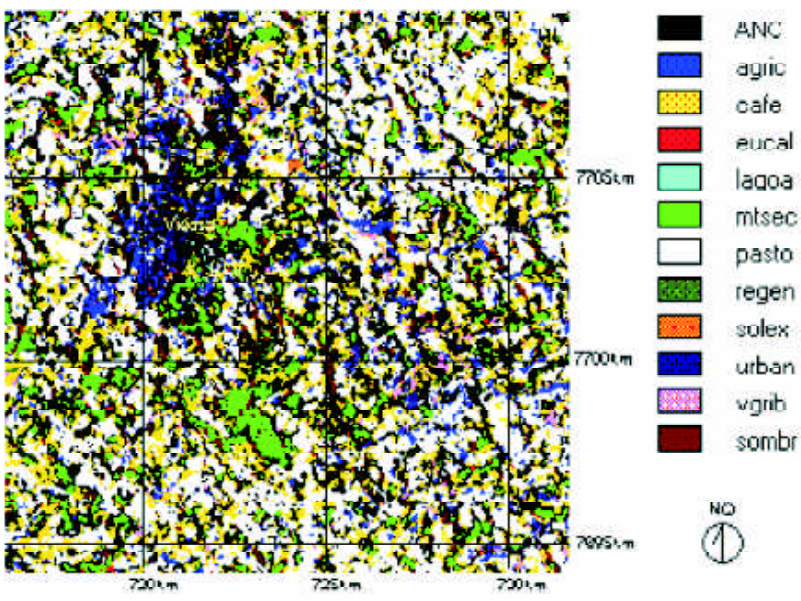

Figura 8 - NPVICA3_6bits pós-processada com filtro de moda/3×3. Grade UTM $(\mathrm{km})$ SAD69. NPVICA3_6bits pós-processing with filter of the mode/3×3. Grade UTM $(\mathrm{km})$ SAD69. 
COSTA, T. C. C. et al. Classificação do uso/cobertura da terra por meio de...

Quadro 10 - Matriz de erros para MAXVER1\%-Linhas, e Pontos de Referência-Colunas. Confusion matrix for MAXVER1\%-rows, and reference points columns.

\begin{tabular}{|c|c|c|c|c|c|c|c|c|c|c|c|c|c|}
\hline & 1 & 2 & 3 & 4 & 5 & 6 & 7 & 8 & 9 & 10 & 11 & Tot. & Erro $\mathrm{C}$ \\
\hline 0 & & & & 1 & & & & & & & & 1 & 1.000 \\
\hline 1 & 18 & 1 & & & & 4 & & & 7 & & & 30 & .400 \\
\hline 2 & & 7 & & & & 2 & 4 & & & 2 & & 15 & .533 \\
\hline 3 & & 1 & 6 & & 13 & & 2 & & & & & 22 & .727 \\
\hline 4 & & & & 4 & & & & & & & & 4 & .000 \\
\hline 5 & & & 2 & & 47 & & 3 & & & & & 52 & .096 \\
\hline 6 & 6 & & & & & 104 & & & 2 & 1 & & 113 & .079 \\
\hline 7 & & 1 & 1 & & 4 & & 11 & & & & & 17 & .352 \\
\hline 8 & & & & & & 2 & & 3 & 1 & & & 6 & .500 \\
\hline 9 & 3 & & & & & & & 1 & 11 & & & 15 & .266 \\
\hline 10 & & & 2 & & 1 & & 1 & & & 6 & & 10 & .400 \\
\hline 11 & & 1 & & 1 & & 3 & & & & & 19 & 24 & .208 \\
\hline Total & 27 & 11 & 11 & 6 & 65 & 115 & 21 & 4 & 21 & 9 & 19 & 309 & \\
\hline Erro 0 & .333 & .363 & .151 & .333 & .276 & .095 & .176 & .250 & .176 & .333 & .000 & & .236 \\
\hline
\end{tabular}

Quadro 11 - Matriz de Erros para NPSKID_6bits- Linhas, e Pontos de Referência- Colunas. Confusion matrix for NPSKID_6bits-rows, and reference pointscolumns.

\begin{tabular}{|c|c|c|c|c|c|c|c|c|c|c|c|c|c|}
\hline & 1 & 2 & 3 & 4 & 5 & 5 & 7 & 8 & 9 & 10 & 11 & Total & Emo \\
\hline 0 & 13 & 4 & 4 & 4 & 15 & 49 & 7 & 4 & 9 & 2 & 10 & 121 & 1.000 \\
\hline 1 & 6 & & & & & 6 & & & 4 & & & 16 & .625 \\
\hline 2 & & 6 & & & & 1 & 1 & & & 3 & & 11 & 454 \\
\hline 3 & & & 4 & & 15 & & 3 & & & & & 22 & 818 \\
\hline 4 & & & & 2 & & & & & & & & 2 & .000 \\
\hline 5 & & 1 & 2 & & 33 & & 2 & & & & & 38 & .131 \\
\hline 6 & 3 & & & & & 59 & & & & 1 & & 63 & .063 \\
\hline 7 & & & & & 2 & & $g$ & & & & & 1 & 200 \\
\hline 8 & 1 & & & & & & & & 1 & & & 2 & 1.000 \\
\hline 9 & 4 & & & & & & & & 7 & & & 11 & 363 \\
\hline 1 & & & 1 & & & & & & & 3 & & 4 & 250 \\
\hline 11 & & & & & & & & & & & 9 & 9 & .000 \\
\hline Tolal & 27 & 11 & 11 & 6 & 65 & 115 & 21 & 4 & 21 & 9 & 19 & 309 & \\
\hline Erro 0 & .777 & .151 & .636 & .666 & .192 & .187 & .619 & 1.00 & .666 & .666 & .526 & & .556 \\
\hline
\end{tabular}

Quadro 12 - Matriz de erros para NPVICA3_6bits- Linhas, e Pontos de Referência- Colunas. Confusion matrix for NPVICA3_6bits-rows, and reference pointscolumns.

\begin{tabular}{|c|c|c|c|c|c|c|c|c|c|c|c|c|c|}
\hline & 1 & 2 & 3 & 4 & 5 & 6 & 7 & 8 & 9 & 10 & 11 & Total & Erro C \\
\hline 0 & 5 & 2 & $\bar{b}$ & 2 & 30 & 10 & 9 & & 7 & & 7 & 78 & 1.000 \\
\hline 1 & 12 & & & & & 7 & & & 7 & & & 26 & .538 \\
\hline 2 & & 8 & & & & 8 & 5 & & & 3 & 1 & 25 & .680 \\
\hline 3 & & & 1 & & 1 & & & & & & & 2 & .500 \\
\hline 4 & & & & 4 & & & & & & & & 4 & .000 \\
\hline 5 & & & & & 31 & & 1 & & & & 5 & 37 & .162 \\
\hline 6 & 6 & & & & & 90 & & & 3 & 1 & & 100 & .100 \\
\hline 7 & & 1 & 2 & & 2 & & 6 & & & & & 11 & .454 \\
\hline 8 & & & & & & & & 3 & 1 & & & 1 & .250 \\
\hline 9 & 4 & & & & & & & 1 & 3 & & & 8 & .625 \\
\hline 10 & & & 2 & & 1 & & & & & 5 & & 8 & .375 \\
\hline 11 & & & & & & & & & & & 6 & 6 & .000 \\
\hline Total & 27 & 11 & 11 & 6 & 65 & 115 & 21 & 4 & 21 & 9 & 19 & 309 & \\
\hline Erro 0 & .555 & .272 & .909 & .333 & .523 & .217 & .711 & 250 & .857 & .111 & .681 & & .153 \\
\hline
\end{tabular}




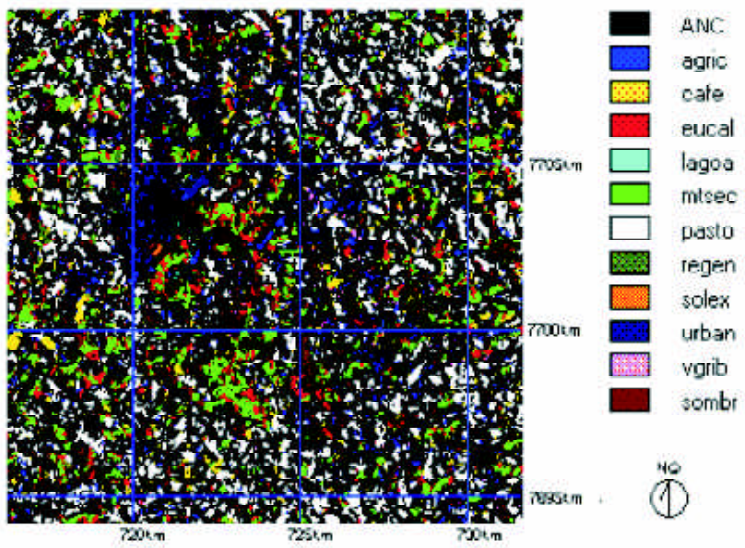

Figura 9 - NPSKID_6bits pós-processada com filtro de moda/3×3. Grade UTM (km) SAD69. NPSKID_6bits pos-processing with filter of the mode/3x3. Grade UTM $(\mathrm{km})$ SAD69.

Skidmore e Turner (1988) alertaram para esta limitação de seu classificador. Em dados SPOT XS, usaram em média 900 pixels por classe, variando de 700 a 2000 pixels, com uma intensidade amostral de treinamento de $1,6 \%\left(n^{0}\right.$ pixels amostrados $/ n^{0}$ pixels da imagem). Dymond (1993) usou intensidades amostrais de 1,95\%; 3,92\%; 2,33\% e 3,4\% em quatro áreas com diferentes condições de iluminação, relevo e cobertura/uso da terra. Usouse neste trabalho uma intensidade amostral de 1,15\% (2996/261121), mas com uma representatividade por classe bem menor em comparação ao mínimo de 700 pixels. Nesta área de estudo, classes como lagoa (LAGOA) e solo exposto (SOLEX) são de baixa ocorrência, e a coleta de mais de 60 pixels para cada classe só foi possível devido à cobertura das fotos aéreas.

Para o classificador de máxima verosimilhança estes tamanhos de amostra são suficientes para representar as classes, porque o ajuste multinormal permite a inclusão de pixels não coletados na amostra, mas estimados pela distribuição. Como esta normalização não ocorre para os classificadores não paramétricos de Skidmore/Turner e "melhorado" por Dymond, eles só são capazes de classificar vetores de pixels que existem na amostra de treinamento, necessitando de uma coleta de amostra de treinamento exagerada, se comparada à exigência do tamanho de amostra para o MAXVER. Já para o NPVIC o $n^{0}$ de bits não altera consideravelmente a exatidão global $(G)$ e a área não classificada (\%ANC), conforme pode ser observado nas figuras 4 e 5 .

Comparando-se as estratégias A e B do NPVIC (figuras 4 e 5), (A) é menos restritiva em classificar um pixel, gerando portanto \%ANC menores. A estratégia (B) gera índices de exatidão $(G)$ maiores, mas de nada adianta classificar uma pequena área da imagem com $100 \%$ de certeza.

A figura 6 mostra o rank dos resultados de $\mathrm{G}$ e \%ANC para todos os classificadores e estratégias aplicados aos dados originais ( 8 bits), com 7 e 6 bits, e o teste $Z$ de significância seqüencial entre os resultados a $1 \%$. A combinação dos melhores resultados de exatidão com as menores percentagens de área não classificada ocorre para o algoritmo MAXVER com opções 0 e $1 \%$. Os maiores níveis de \%ANC estão representados pelos 
algoritmos NPSKID, NPSKIDYM e NPVICB3. E os menores índices de exatidão (G) estão representados pelos algoritmos NPVICA1 e NPVICDYMA1.

Três classificações de Uso/Cobertura da Terra e respectivas matrizes de erros (quadros 10,11 e 12) foram apresentadas como exemplo:

A primeira (figura 7 ) foi obtida pelo MAXVER, com exatidão de $76,62 \%$, sem limitações para classificar área da imagem. Com a decisão de classificar pixels até $1 \%$ de significância, não classificou apenas $1,73 \%$ da cena.

A segunda (figura 8) foi obtida com a estratégia A do NPVIC com exigência de interseção nas três Bandas, com 6 bits. Esta foi o melhor resultado do NPVIC, mas não satisfatório, com exatidão de $73,16 \%$ e não classificando $28,03 \%$ da imagem.

A terceira (figura 9) foi o melhor resultado do NPSKID, com 6 bits, não classificando $51,7 \%$ da imagem, com $\mathrm{G}$ de $72,87 \%$.

\section{CONCLUSÕES}

O algoritmo Não Paramétrico de Skidmore/Turner, para tamanhos de amostra de treinamento suficientes ao classificador de máxima verossimilhança, produz classificações de baixa qualidade. Aplicado em dados originais, classifica um pouco mais do que as próprias amostras de treinamento, e aplicado a dados comprimidos, perde exatidão, e mesmo assim não atinge níveis aceitáveis de área não classificada.

O algoritmo Não Paramétrico desenvolvido neste trabalho (NPVIC) permite reduzir a área não classificada em uma imagem, quando comparado ao algoritmo Não Paramétrico de Skidmore/Turner, mas não de forma satisfatória, pois para classificar toda a área gera imagens de menor exatidão, comparado ao classificador de máxima verossimilhança (MAXVER).

A normalização proposta por Dymond apresenta sensivel melhora no algoritmo de Skidmore/Turner. Esta estratégia aplicada ao NPVIC reduziu a área não classificada, mas com relação à exatidão tornou-se instável, com piores resultados em sua maioria, quando comparado ao NPVIC.

O MAXVER, comparado a estas alternativas não paramétricas, é um algoritmo superior em: esforço amostral de treinamento, e em exatidão para estas condições. Suas vantagens estão associadas à correção da representatividade das amostras de treinamento, por meio da multinormalização das classes.

\section{AGRADECIMENTOS}

Os autores agradecem ao Instituto Nacional de Pesquisas Espaciais - INPE, pela doação da cena utilizada neste estudo. Os algoritmos desenvolvidos neste trabalho podem ser solicitados ao primeiro autor.

\section{REFERÊNCIAS}

CORTIJO F.J. \& LA BLANCA N.P. 1997. A comparative study of some non-parametric spectral classifiers. Applications to problems with high-overlapping training sets. International Journal of Remote Sensing, 18: 1259-1275.

COSTA T.C.C. 1999. Avaliação de estratégias para classificação de imagens orbitais em mapeamento de uso e cobertura da terra. Universidade Federal de Viçosa, Minas Gerais, Tese de Doutorado, 109 p.

DYMOND J.R. 1993. An improved Skidmore/Turner classifier. Photogrammetric Engineering and Remote Sensing, 59: 623-626.

GONG P. \& DUNLOP J.D. 1991. Comments on the Skidmore and Turner supervised nonparametric 
COSTA, T. C. C. et al. Classificação do uso/cobertura da terra por meio de...

classifier. Photogrammetric Engineering and Remote Sensing, 57: 1311-1313.

JENSEN, J.R. 1996. Introductory digital image processing - a remote sensing perspective. Englewood Cliffs, Prentice-Hall, $379 p$.

KLOER B.R. 1994. Hybrid parametric/non-parametric image classification. In: ASPRS/ACSM Annual Convention E Exposition Technical Papers, 1, Nevada. Proceedings... Nevada, 1:307-316.

LOWELL K.E. 1989. A probabilistic modification of the decision rule in the Skidmore/Turner supervised nonparametric classifier. Photogrammetric Engineering and Remote Sensing, 55: 897-900.

MA Z. \& REDMOND R.L. 1995. Tau coefficients for accuracy assessment of classification of remote sensing data. Photogrammetric Engineer and Remote Sensing, 61:453-439.

SKIDMORE A.K. \& TURNER B.J. 1988. Forest mapping accuracies are improved using a supervised nonparametric classifier with SPOT data. Photogrammetric Engineering and Remote Sensing, 54: $1415-1421$.

SWAIN P.H. \& DAVIS S.M. 1978. Remote sensing: the quantitative approach. New York, McGrawHill, 396 p.

Recebido em 10 maio 2004

Aceito em 10 maio 2005 